\section{LETHAL SINGLE VEHICLE ACCIDENTS OF ESC FITTED PASSENGER CARS}

1,2Tapio Koisaari, ${ }^{3}$ Timo Tervo, ${ }^{2}$ Niina Sihvola. ${ }^{1}$ Department of Engineering Design and Production, Aalto University, Aalto, Finland; '2Finnish Motor Insurers' Centre, Helsinki, Finland; ${ }^{3}$ Department of Ophthalmology, University of Helsinki and Helsinki University Hospital, Helsinki, Finland

\subsection{6/injuryprev-2016-042156.185}

Background Fatal single vehicle accidents (SVA) which involved ESC fitted passenger cars were examined in order to disclose the immediate and background risk factors for the accidents as well as the validity of ESC function.

Methods The registry of Finnish Road Accident (FRA) Investigation Teams (RAIT) was analysed and all fatal passenger car accidents during the years 2009-13 (5 years) were included. SVAs $(\mathrm{N}=351)$ were subjected to more detailed analysis. Suicide crashes $(N=13)$ and disease attacks $(N=102)$ were recognised but ruled out from the final results because ESC has no saving potential in such accidents.

Results ESC fitted vehicles were involved in SVAs less often $(P=0.001)$ than their calculated exposure would suggest and they were quite rare. The most common immediate risk factor in ESC SVAs was a steering error $(56 \%, \mathrm{~N}=10)$ but unlike in the non-ESC SVAs it did not lead to loss of control of the vehicle.

The common background risk factors in ESC SVAs were substance abuse $(56 \%, \mathrm{~N}=10)$, tiredness $(56 \%, \mathrm{~N}=10)$ and speeding $(56 \%, \mathrm{~N}=10)$. Tiredness was more common $(\mathrm{P}=0.01)$ than in non-ESC SVAs and in half of the accidents it occurred together with substance abuse.

In lethal ESC SVAs the vehicle experienced multiple collisions (2.4 on average) at relatively high speed (on the average $93 \mathrm{~km} / \mathrm{h}$ before first impact). This is a challenge to both passive and active safety systems. Also in ESC SVAs the seat belt use was low; nine $(47 \%)$ of the killed occupants were unbelted. Seat belt use would have saved six (\%) of them.

Conclusions Based on this study, ESC operates as designed in SVAs and no operational failures were found. Further research on driver fitness is required to improve active safety of the passenger cars. Vehicle systems should force everyone to use seat belt and the strength of roof structures should be improved.

\section{U.S. RECREATIONAL OFF-HIGHWAY VEHICLE CRASHES; AN EMERGING HEALTH AND SAFETY CONCERN}

Gerene Denning. Charles Jennissen. Department of Emergency Medicine, University of lowa Carver College of Medicine, USA

\subsection{6/injuryprev-2016-042156.186}

Background All-terrain vehicle (ATV) crashes have long been recognised as a public health concern, with $>800$ deaths and more than 400,000 injuries each year in the U.S. alone. Recreational off-highway vehicles (ROVs) have more recently been growing in popularity, but little is currently known about the impact of their use. Our objective was to determine the epidemiology, mechanisms and risk factors for ROV crashes and injuries.

Methods Descriptive and comparative analyses were performed using data from the Consumer Product Safety Commission ROV database (2003-2011).

Results The database contained 428 crashes (899 occupants). Occupants were $68 \%$ male, $31 \%<16$ years old, and $52 \%$ passengers. Five percent of occupants were helmeted and $27 \%$ were restrained. Half of the operators were positive for alcohol and
$9 \%$ for drugs. For crashes, 53\% were on roads, $12 \%$ were occupational, and $63 \%$ occurred at speeds $<20 \mathrm{mph}$. The primary crash mechanism was a non-collision event, predominately rollovers with $95 \%$ being sideway rollovers and $81 \%$ of sideway rollovers occurring during a turn. Many crashes occurred on dry surfaces $(84 \%)$, in good weather $(94 \%)$, and/or on flat surfaces $(63 \%)$ or gentle slopes $(15 \%)$. For injuries, 22\% were moderate to severe and $36 \%$ were fatal. Fifteen percent of occupants were partially ejected and 51\% were fully ejected. Fatal crashes were 4 times more likely to involve an operator's death than a passenger's, rollovers increased the odds of being ejected by 13 -fold, and both ejection and being pinned by the vehicle increased the likelihood of death by over 10 -fold and 20 -fold, respectively.

Conclusions These data provide important insights into ROV use and related crashes. Although likely to be more stable than ATVs, ROV rollovers, including at low speeds, appear to be the major crash mechanism, most often in a turn. Being unrestrained in a rollover with subsequent ejection and pinning by the ROV were the most significant risk factors for severe injury and death.

\section{EXPERT-DEVELOPED ICD-AIS MAP FOR MEASURING SERIOUS ROAD TRAFFIC INJURIES}

${ }^{1,2}$ Jo Barnes, ${ }^{1,3}$ Jan Price, ${ }^{1}$ Patrick Gillich, ${ }^{3}$ Amy Brammer, ${ }^{3}$ Christine Read-Allsopp, ${ }^{3}$ Donna Nayduch, ${ }^{3}$ Katherine Baus, ${ }^{3}$ Kathy Cookman, ${ }^{3}$ Kathryn Loftis, ${ }^{3}$ Lauren Jones, ${ }^{3}$ Maureen Brennan, ${ }^{3}$ Patsye Stanley, ${ }^{3}$ Patricia St Germain, ${ }^{3}$ Vickie Graymire. 'Association for the Advancement in Automotive Medicine (AAAM), USA; ${ }^{2}$ Loughborough University, UKi ${ }^{3}$ Expert Panel Group

\subsection{6/injuryprev-2016-042156.187}

Background The problem of serious road injury has not decreased in comparison to the drop in fatal road injuries. Unlike the standard definition adopted for fatal road injuries, serious injury has not been as well defined in Europe. However in 2012 the High Level Group on road safety agreed to the defining of serious injury in EU countries as Maximum Abbreviated Injury Scale $3+($ MAIS3 +$)$. The AAAM was commissioned by the Transport and Mobility, Road Safety Unit of the European Commission to develop a MAIS3 + serious injury map from the International Classification of Diseases (ICD-9 and ICD-10) to the Abbreviated Injury Scale (AIS 2005 Update 2008).

Methods The study involved an expert panel comprising ICD coding experts and certified AIS specialists to map ICD-9-CM and ICD-10-CM codes to the AIS 2005 Update 2008 identifying the following levels of severity: MAIS3 + injury, MAIS 1 and 2 and 'no map' cases. Rules were invoked based on the AIS principles of coding practice to ensure the MAIS descriptors were a true reflection of how an injury descriptor in ICD would be coded manually to the AIS by a certified AIS specialist.

Results Overall for the injury-based codes of ICD-9-CM, 781 codes $(31 \%)$ were mapped to a MAIS severity at the level of MAIS3 +, 1297 codes (52\%) were mapped at the level of MAIS1 or 2 and 426 codes (17\%) could not be mapped. For the much larger set of injury-based codes of ICD-10-CM, 2323 codes (14\%) were mapped to a MAIS severity at the level of MAIS3+, 9700 codes $(59 \%)$ at the level of MAIS1 or 2, and $4485(27 \%)$ could not be mapped.

Conclusions The application of the AAAM's expert derived AIS ICD map is a solid foundation for identifying serious injury at MAIS3 + for road traffic injuries. This AIS-ICD map offers a comprehensive approach to serious road injury definition for the EU and will enable countries to set measureable road safety 